\title{
A tale of many electrons
}

\author{
Density functional theory, invented half a century ago, now supplies one of the most convenient and \\ popular shortcuts for dealing with systems of many electrons. It was born in a fertile period when \\ theoretical physics stretched from abstruse quantum field theory to practical electrical engineering.
}

It's often pointed out that quantum theory is not just a source of counterintuitive mystery but also an extraordinarily effective intellectual foundation for engineering. It supplies the theoretical basis for the transistor and superconductor, for understanding molecular interactions relevant from mineralogy to biology, and for describing the basic properties of all matter, from superhard alloys to high-energy plasmas. But popular accounts of quantum physics rarely pay more than lip service to this utilitarian virtue - there is little discussion of what it took to turn the ideas of Bohr, Heisenberg and Schrödinger into a theory that works at an everyday level.

One of the milestones in that endeavour occurred 50 years ago, when Pierre Hohenberg and Walter Kohn published a paper ${ }^{1}$ that laid the foundations of density functional theory (DFT). This provided a tool for transforming the fiendishly complicated Schrödinger equation of a many-body system such as the atomic lattice of a solid into a mathematically tractable problem that enables the prediction of properties such as structure and electrical conductivity. The milieu in which this advance was formulated was rich and fertile, and from the distance of five decades it is hard not to idealize it as a golden age in which scientists could still see through the walls that now threaten to isolate disciplines. Kohn, exiled from his native Austria as a young Jewish boy during the Nazi era and educated in Canada, was located at the heart of this nexus. Schooled in quantum physics by Julian Schwinger at Harvard among peers including Philip Anderson, Rolf Landauer and Joaquin Luttinger, he was also familiar with the challenges of tangible materials systems such as semiconductors and alloys. In the mid-1950s Kohn worked as a consultant at Bell Labs, where the work of John Bardeen, Walter Brattain and William Shockley on transistors a few years earlier had generated a focus on the solid-state theory of semiconductors. And his ground-breaking paper with Hohenberg came from research on alloys at the Ecole Normale Supérieure in Paris, hosted by Philippe Nozières.

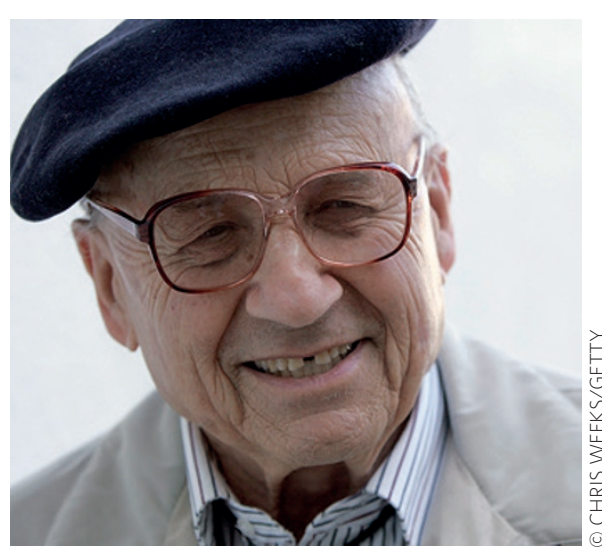

simple that he couldn't believe it hadn't been noticed before. So he asked Hohenberg, a postdoc in Nozières's lab, to help. Together the pair formulated a rigorous proof of the conjecture for the case of an inhomogeneous electron gas; since their 1964 paper, several other proofs have been found. That paper was formal and understated to the point of desiccation, and one needed to pay it close attention to see how remarkable the result was. The initial response was muted, and Hohenberg moved subsequently into other areas, such as hydrodynamics, phase transitions and pattern formation.

Kohn, however, went on to develop the idea into a practical method for calculating the electronic ground states of molecules and solids, working in particular with Hong Kong-born postdoc Lu-Jeu Sham. Their crucial paper ${ }^{3}$ was much more explicit about the potential of this approach as an approximation for calculating real materials properties of solids, such as cohesive energies and elastic constants, from quantum principles. It is now one of the most highly cited papers in all of physics, but was an example of a 'sleeper': still the community took some time to wake up to what was on offer. Not until the work of John Pople in the early 1990s did chemists begin to appreciate that DFT could offer a simple and convenient way to calculate electronic structures. It was that work that led to the 1998 Nobel Prize in Chemistry for Pople and Kohn - incongruous for someone so immersed in physics.

Zangwill argues that DFT defies the common belief that important theories reflect the zeitgeist: it was an idea that was not in the air at all at its inception, and, says Zangwill, "might be unknown today if Kohn had not created it in the mid-1960s." Clearly that's impossible to prove. But there's no mistaking the debt that materials and molecular scientists owe to Kohn's insight, and so if Zangwill is right, all the more reason to ask if we still create the right sort of environments for such fertile ideas to germinate.

References

1. Hohenberg, P. \& Kohn, W. Phys. Rev. 136, B864-B871 (1964).

2. Zangwill, A. Preprint at http://arxiv.org/abs/1403.5164 (2014).

3. Kohn, W. \& Sham, L. J. Phys. Rev. 140, A1133-A1138 (1965). 